\title{
Fate tracing of mature hepatocytes in mouse liver homeostasis and regeneration
}

\author{
Yann Malato,, 1,2 Syed Naqvi, ${ }^{1,3}$ Nina Schürmann, ${ }^{4}$ Raymond Ng, ${ }^{1,2}$ Bruce Wang, ${ }^{5}$ \\ Joan Zape, ${ }^{1}$ Mark A. Kay, ${ }^{6}$ Dirk Grimm, ${ }^{4}$ and Holger Willenbring ${ }^{1,2,3}$
}

\begin{abstract}
${ }^{1}$ Eli and Edythe Broad Center of Regeneration Medicine and Stem Cell Research, ${ }^{2}$ Department of Surgery, Division of Transplantation, and ${ }^{3}$ Liver Center, UCSF, San Francisco, California, USA. ${ }^{4}$ Cluster of Excellence CellNetworks, Department of Infectious Diseases/Virology, Heidelberg University, Heidelberg, Germany. ${ }^{5}$ Department of Developmental Biology and ${ }^{6}$ Department of Pediatrics and Genetics, Stanford University, Stanford, California, USA.
\end{abstract}

\begin{abstract}
Recent evidence has contradicted the prevailing view that homeostasis and regeneration of the adult liver are mediated by self duplication of lineage-restricted hepatocytes and biliary epithelial cells. These new data suggest that liver progenitor cells do not function solely as a backup system in chronic liver injury; rather, they also produce hepatocytes after acute injury and are in fact the main source of new hepatocytes during normal hepatocyte turnover. In addition, other evidence suggests that hepatocytes are capable of lineage conversion, acting as precursors of biliary epithelial cells during biliary injury. To test these concepts, we generated a hepatocyte fate-tracing model based on timed and specific Cre recombinase expression and marker gene activation in all hepatocytes of adult Rosa26 reporter mice with an adenoassociated viral vector. We found that newly formed hepatocytes derived from preexisting hepatocytes in the normal liver and that liver progenitor cells contributed minimally to acute hepatocyte regeneration. Further, we found no evidence that biliary injury induced conversion of hepatocytes into biliary epithelial cells. These results therefore restore the previously prevailing paradigms of liver homeostasis and regeneration. In addition, our new vector system will be a valuable tool for timed, efficient, and specific loop out of floxed sequences in hepatocytes.
\end{abstract}

\section{Introduction}

The liver is currently believed to be unique among adult mammalian organs in that normal turnover or homeostasis of its fully differentiated parenchymal cells (hepatocytes and biliary epithelial cells) is the result of self duplication. In accordance with this view, the ability of the liver to rapidly regenerate after tissue injury or loss rests on the stem cell-like proliferative potential of all residual parenchymal cells and not on that of an elite subpopulation (1-8). However, if hepatocytes or biliary epithelial cells lose the ability to proliferate, such as in chronic injury states, liver progenitor cells are activated. Liver progenitor cells reside in or around bile ducts within periportal areas and, depending on the nature of the injury, give rise to hepatocytes or biliary epithelial cells (9-11).

Whether liver progenitor cells contribute to maintaining the hepatocyte mass during homeostasis of the normal adult liver has been the focus of a long-standing debate. Findings made in rats many years ago (12), but recently confirmed in humans (13), suggest that new hepatocytes derive from periportally localized liver progenitor cells and migrate progressively toward the central vein. This "streaming liver" model has been contradicted by several studies showing that hepatocytes form small, presumably clonal, clusters throughout the liver parenchyma in adult rats (14) and mice (15) over time. In addition, liver progenitor cells were previously only identified in injured livers in humans (16). However, more recent reports suggest that the normal adult human liver contains a large number of liver progenitor cells that may contribute to liver homeostasis (17). In fact, Furuyama et al. reported recently that liver progenitor cells residing in bile ducts are the predominant source of new hepatocytes in mouse liver homeostasis and afford near-complete turnover of the hepatocyte mass within 6 months (18). They also showed that liver progenitor cells give

Conflict of interest: The authors have declared that no conflict of interest exists. Citation for this article: J Clin Invest. 2011;121(12):4850-4860. doi:10.1172/JCI59261. rise to hepatocytes after two-thirds partial hepatectomy $(2 / 3 \mathrm{PH})$ and carbon tetrachloride $(\mathrm{CCl} 4)$ intoxication, both of which are experimental models believed to trigger hepatocyte regeneration only by self duplication (19-23).

Other recent findings challenge the current belief that new biliary epithelial cells derive only from 2 sources: mature biliary epithelial cells or, if their ability to proliferate is impaired, liver progenitor cells $(24,25)$. The new findings suggest that hepatocytes can also contribute to the formation of new biliary epithelial cells in states of biliary injury, in particular if the biliary injury is so severe that the proliferation of both biliary epithelial cells and liver progenitor cells is blocked (26-29). This lineage conversion is preceded by activation of biliary markers, such as cytokeratin 19 (CK19) in periportal hepatocytes, which can also be observed in humans with chronic biliary diseases $(30,31)$. The newly expressed genes include the biliary-specific transcription factor hepatocyte nuclear factor $1 \beta$ (HNF $1 \beta$ ) (29), a finding that recalls recent reports showing that overexpression of cell type-specific transcription factors can induce lineage conversion in several tissues in mice (32).

These findings suggest an overlooked role of liver progenitor cells in normal hepatocyte turnover and regeneration, and raise the intriguing possibility that hepatocytes can assume a different cellular identity and function in response to injury. Because these findings call for correcting or amending our current understanding of liver homeostasis and regeneration (33), we decided to test them using a different experimental approach. Instead of using in situ fate tracing of liver progenitor cells (18) or hepatocyte transplantation (26-29), as in the original studies, we established in situ fate tracing of hepatocytes to address 2 questions. First, are new hepatocytes produced during liver homeostasis and in response to acute hepatocyte injury or loss derived from cells other than hepatocytes? Second, does biliary injury induce conversion of hepatocytes into biliary epithelial cells? 
A

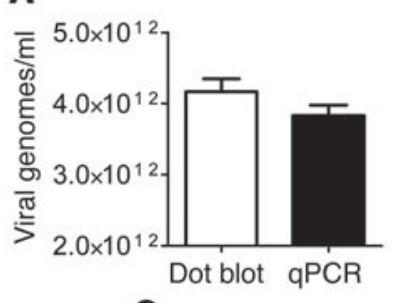

C
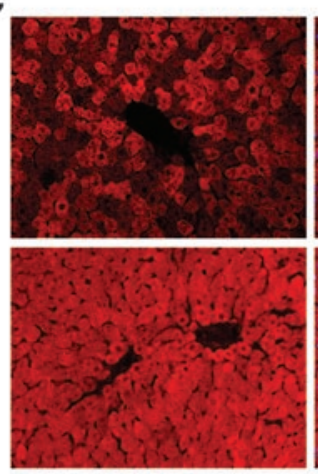

EYFP

D

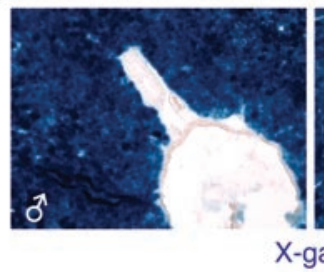

B
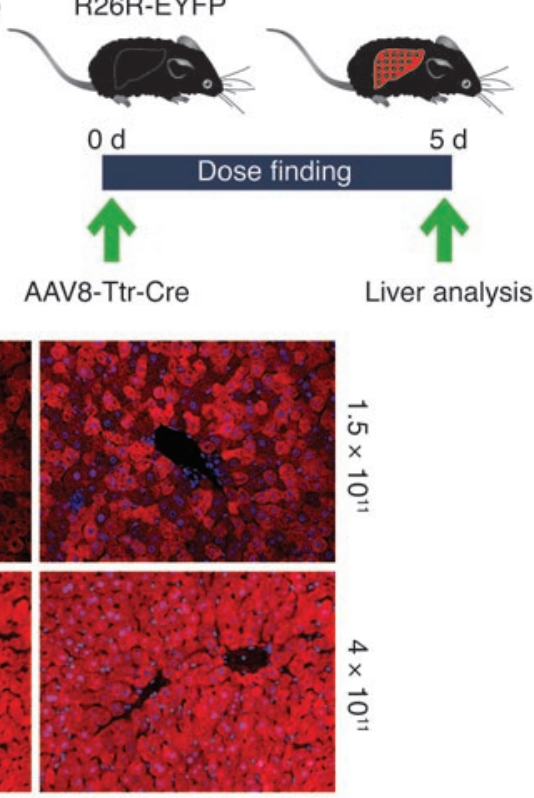

EYFP + DAPI

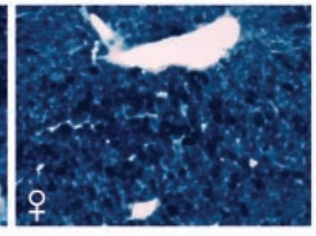

X-gal

\section{Figure 1}

Rapid marker gene activation in all hepatocytes of adult R26R-EYFP and R26R mice injected with AAV8-Ttr-Cre. (A) Results of AAV8-Ttr-Cre vector titration by dot blot and qPCR. The titer was approximately $4 \times 10^{12}$ viral genomes $/ \mathrm{ml}$ in both dot blot and qPCR analysis (Supplemental Figure 1, $A$ and $B$ ). Data represent mean \pm SEM. (B) Dose-finding strategy. Livers were analyzed by immunostaining for EYFP 5 days after injection of different AAV8-Ttr-Cre doses into the tail veins of adult R26R-EYFP mice. (C) $1.5 \times 10^{11}$ viral genomes were not sufficient to activate EYFP expression (red) in all hepatocytes. $4 \times 10^{11}$ viral genomes activated EYFP expression in all hepatocytes. Nuclei were stained with DAPI (blue). (D) X-gal staining of livers of male and female R26R mice 48 hours after injection of $4 \times 10^{11}$ viral genomes shows $\beta$-gal expression (blue) in all hepatocytes. 15 liver sections from 3 mice were analyzed for each experiment. Original magnification, $\times 200$.
Answering these questions required specific labeling of hepatocytes and their progeny in the adult mouse liver. Moreover, determining the origin of newly formed hepatocytes by hepatocyte fate tracing required labeling of all hepatocytes. A commonly used approach to following cell fate is to introduce an inducible cell type-specific Cre recombinase allele into Rosa26 reporter (R26R) mice, which carry a marker gene inactivated by a floxed stop codon in the ubiquitously expressed Rosa26 locus (34). Induction of Cre in such mice leads to stable expression of the marker gene in the targeted cell type and the progeny thereof. Because mice currently available for temporally controlled Cre expression in hepatocytes appear to lack the efficiency or specificity needed for hepatocyte fate tracing (35-41), we generated an adenoassociated virus (AAV) vector that expresses Cre and loops out floxed sequences in all hepatocytes, but not in other liver cell types, of adult mice within 48 hours after intravenous injection.

\section{Results}

Efficient and specific loop out of floxed sequences in hepatocytes of adult mice. We built the new vector, designated AAV8-Ttr-Cre, on a double-stranded AAV backbone that affords more rapid and efficient transgene expression than conventional backbones because it bypasses the need for conversion from a single-stranded to a double-stranded state after transduction (42). To optimize loop-out efficiency, we used a Cre linked to a nuclear translocation signal (43). To restrict Cre expression to hepatocytes, we used a hepatocyte-specific transthyretin ( $\mathrm{Ttr}$ ) promoter and pseudotyped the vector genomes with capsids from AAV8, a serotype that affords efficient and specific transgene expression in hepatocytes in vivo at low vector doses (44). We determined the vector titer by dot blot and quantitative PCR (qPCR) (Figure 1A and Supplemental Figure 1, $\mathrm{A}$ and $\mathrm{B}$; supplemental material available online with this article; doi:10.1172/JCI59261DS1).

To identify the AAV8-Ttr-Cre dose needed for loop out of the floxed stop codon and marker activation in all hepatocytes, we intravenously injected increasing vector doses into 8- to 10-week-old R26R mice (Figure 1B). We used 2 types of R26R mice, the original type that conditionally expresses $\beta$-gal detectable by X-gal staining (34) and a variant in which $\beta$-gal was replaced with enhanced yellow fluorescent protein (EYFP) detectable by immunostaining and fluorescent microscopy (45). All mice were on a pure C57BL/6 background and bred and kept under barrier conditions. We analyzed their livers 5 days after AAV8-Ttr-Cre injection and found that $4 \times 10^{11}$ viral genomes were sufficient to activate EYFP expression in all hepatocytes of R26R-EYFP mice (Figure 1C). In fact, the marker gene was already expressed at 48 hours after AAV8-TtrCre injection in both R26R-EYFP and R26R mice, regardless of whether they were male or female (Figure $1 \mathrm{D}$ and data not shown). AAV8 transduction itself did not cause marker gene activation, as is evident from the absence of X-gal-positive hepatocytes in R26R mice injected with $4 \times 10^{11}$ viral genomes of AAV8-Ttr-control, a sibling vector of AAV8-Ttr-Cre, which expresses EYFP instead of Cre (Supplemental Figure 2).

To rule out toxicity of AAV8-Ttr-Cre, which could cause loss of transduced hepatocytes and confound fate-tracing analysis, we analyzed blood and livers of R26R-EYFP mice injected with $4 \times 10^{11}$ 
A

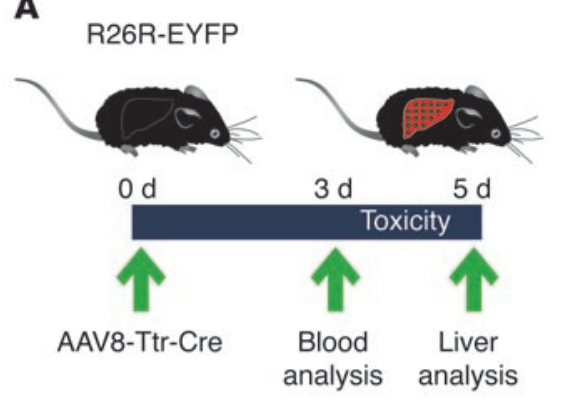

B
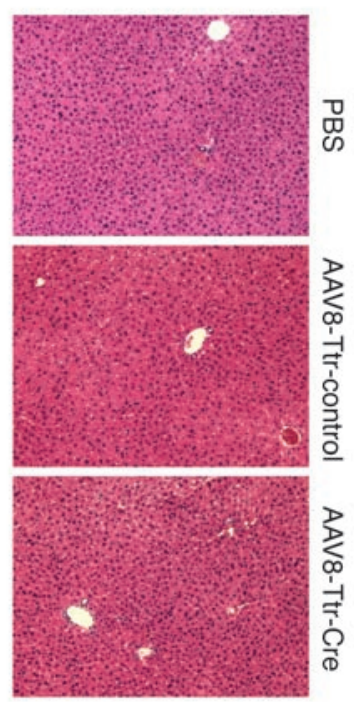

C

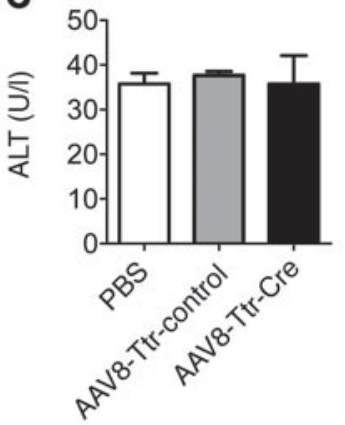

D
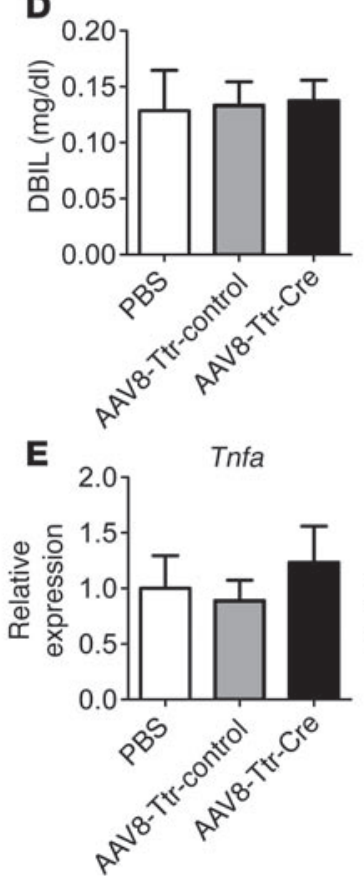
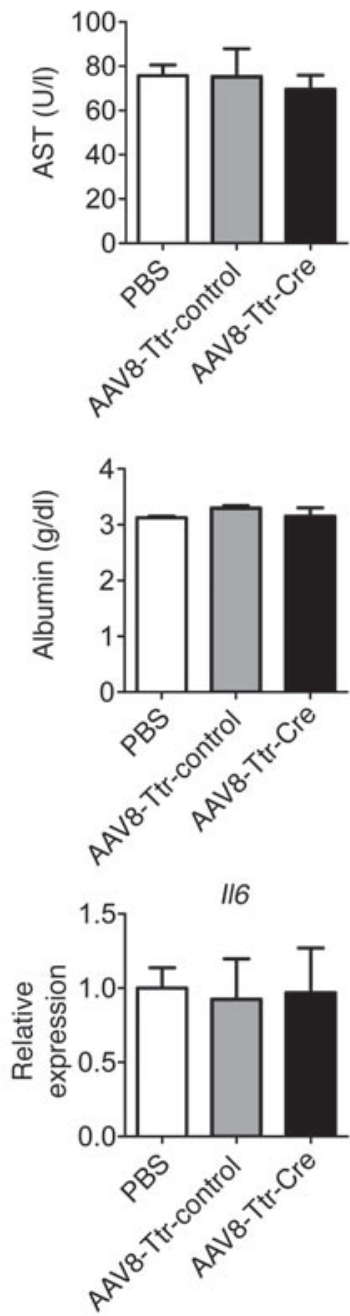

Figure 2

AAV8-Ttr-Cre is not hepatotoxic. (A) Toxicity testing. Blood and livers of adult R26R-EYFP mice were analyzed 3 and 5 days after injection of $4 \times 10^{11}$ viral genomes of AAV8-Ttr-Cre, AAV8-Ttr-control, or PBS, respectively. (B) $H \& E$ staining shows normal liver histologies 5 days after virus and PBS injection. Original magnification, $\times 100.12$ liver sections from 4 mice were analyzed per group. (C and D) Normal ALT and AST transaminase, direct bilirubin (DBIL), and albumin blood levels show that hepatocytes are not injured (C) and function normally (D) in AAV8-Ttr-Cre-injected mice. (E) qRT-PCR analysis shows that the inflammationassociated genes Tnfa and $I / 6$ are not induced in livers of AAV8-TtrCre-injected mice. 4 mice were analyzed per group. Data represent mean \pm SEM. viral genomes of AAV8-Ttr-Cre and compared the results with those from mice injected with AAV8-Ttr-control or PBS (Figure 2, A-E, and Supplemental Figure 3, A-C). We found that AAV8-TtrCre-injected mice were completely normal, including markers of hepatocyte function, injury, or apoptosis and liver inflammation or fibrosis. Moreover, AAV8-Ttr-Cre injection did not induce hepatocyte proliferation, providing additional evidence for undisturbed liver homeostasis (Supplemental Figure 3D).

Next, we investigated whether AAV8-Ttr-Cre loops out floxed sequences specifically in hepatocytes or also in other liver cell types. Coexpression of cell type-specific markers and EYFP, but not $\beta$-gal, can be readily detected in the liver by immunostaining. Therefore, we used exclusively R26R-EYFP mice from this point on. First, by coimmunostaining for major urinary protein (MUP) or HNF $4 \alpha$, we confirmed that all hepatocytes activate EYFP in response to AAV8-Ttr-Cre injection (Figure 3A and Supplemental Figure 4A). MUP is expressed only in mature hepatocytes (46), whereas HNF $4 \alpha$ is also detectable in liver progenitor cells committed to hepatocyte differentiation $(9,10)$. Then we excluded that AAV8-Ttr-Cre activates EYFP expression in biliary epithelial cells or liver progenitor cells. Both of these cell types express the biliary marker CK19 (10, $11,18)$. To establish sensitive immunostaining of cells that express CK19 together with EYFP, we used R26R-EYFP mice carrying a Cre transgene under the transcriptional control of albumin promoter and enhancer sequences (Alb-Cre) (47). Previous reports suggested that Alb-Cre affords loop out of floxed sequences in biliary cells in adult mice, not because it is aberrantly expressed in these cells, but because it is expressed in bipotent fetal liver progenitor cells that pass on the genome modification to both hepatocytes and biliary cells $(48,49)$. In accordance with these reports, we found that all biliary cells were positive for both EYFP and CK19 in adult Alb-Cre, R26R-EYFP mice (Figure 3B). In contrast, we did not find any cells that expressed both EYFP and CK19 in livers of R26R-EYFP mice injected with AAV8-Ttr-Cre (Figure 3C). We confirmed this result by excluding AAV8-Ttr-Cre-mediated loop out of the floxed stop codon in the R26R-EYFP gene in biliary epithelial cells and liver progenitor cells isolated by FACS (Supplemental Figure 4, B-D). In addition, we determined that liver macrophages, stellate cells, and endothelial cells, as identified by F4/80, desmin/ $\alpha$-SMA/glial fibrillary acidic protein (GFAP), and Griffonia simplicifolia lectin I (isolectin $\mathrm{B}_{4}$ ) labeling (50-53), were negative for EYFP (Figure 3, D-F). These results show that AAV8-Ttr-Cre activates EYFP expression in livers of R26R-EYFP mice, specifically in hepatocytes.

The origin of new hepatocytes in liver homeostasis. To resolve the contradiction between emerging evidence suggesting that liver progenitor cells play a critical role in liver homeostasis $(13,17,18)$ 
A
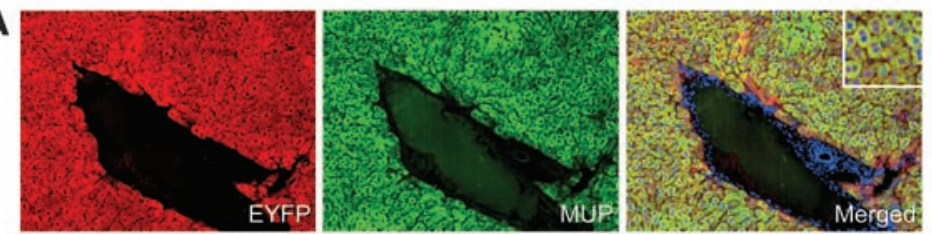

B
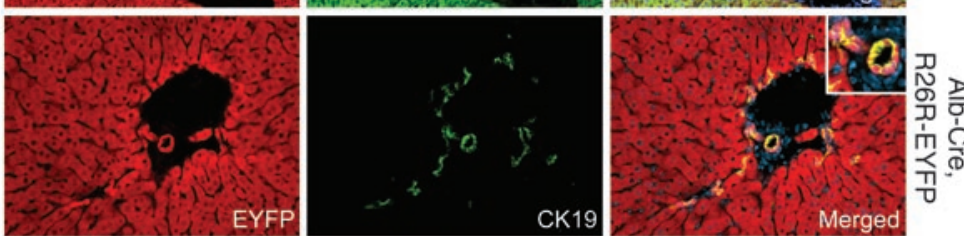

C
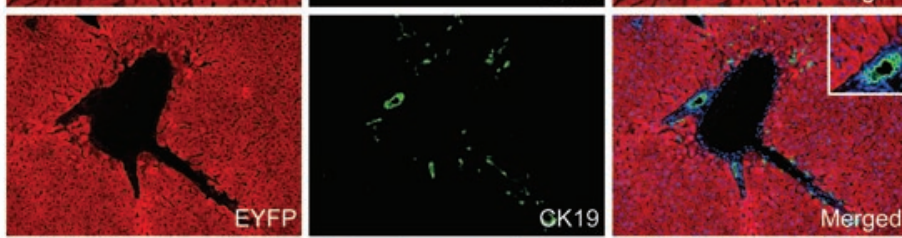

D
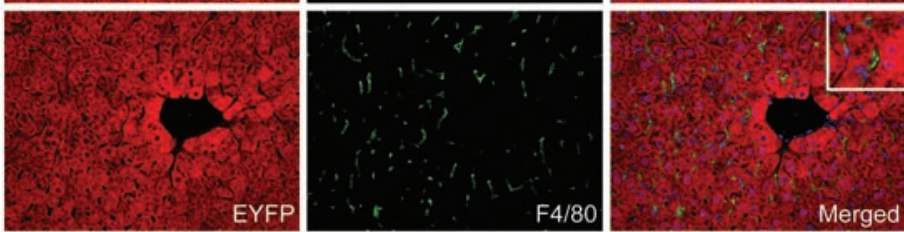

E
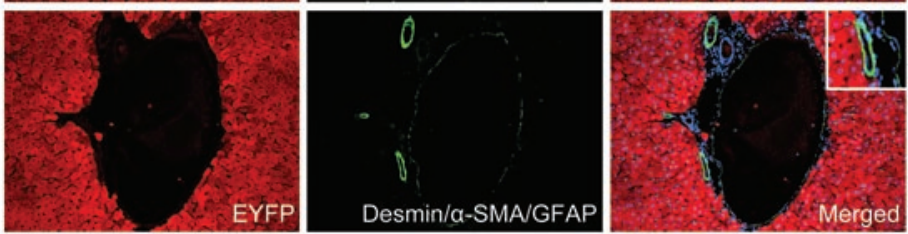

F
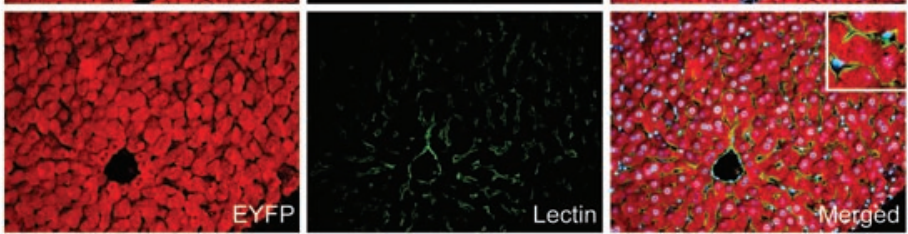

Figure 3

Hepatocyte-specific activation of EYFP expression in adult R26R-EYFP mice injected with AAV8-Ttr-Cre. Livers were coimmunostained for EYFP (red) and cell type-specific markers (green) 5 days after injection of $4 \times 10^{11}$ viral genomes of AAV8-Ttr-Cre. Double-positive cells appear yellow. (A) All MUP-positive cells are also EYFP positive, which confirms that AAV8-Ttr-Cre loops out floxed sequences in all hepatocytes of adult mice. (B) In Alb-Cre, R26R-EYFP control mice, not only all hepatocytes, but also all CK19-positive biliary cells, express EYFP. (C) All cells positive for CK19 are EYFP negative, which shows that AAV8-Ttr-Cre does not loop out floxed sequences in biliary epithelial cells and liver progenitor cells. (D-F) All cells positive for F4/80 (D), desmin/ $\alpha$-SMA/GFAP (E), or isolectin B 4 ("Lectin") (F) are EYFP negative, which shows that AAV8-Ttr-Cre does not loop out floxed sequences in liver macrophages, stellate cells, or sinusoidal, portal vein, and central vein endothelial cells. Nuclei were stained with DAPI (blue). Original magnification, $\times 100$, insets $\times 200(\mathbf{A}, \mathbf{C}$, and $\mathbf{E}) ; \times 200$, insets $\times 400(\mathbf{B}, \mathbf{D}$, and $\mathbf{F}) .20$ liver sections from 4 mice were analyzed for each experiment.

and the conventional view of hepatocyte self duplication as the principal mechanism (14-16), we decided to quantify the hepatocytes newly generated during liver homeostasis that are not derived from preexisting hepatocytes. For this purpose, we established stable expression of EYFP in every hepatocyte of adult R26R-EYFP mice by injecting them with $4 \times 10^{11}$ viral genomes of AAV8-Ttr-Cre. Because all cells derived from hepatocytes also sta- bly express EYFP in these mice, we designated them our hepatocyte fate-tracing model.

Hepatocyte turnover in the normal adult liver is slow - the life span of a hepatocyte ranges from 200 to 400 days in mice (54) and rats (55). Therefore, we waited until 3 or 6 months after AAV8-Ttr-Cre injection before analyzing the mice to allow turnover of at least one-fourth or one-half of the hepatocyte mass, respectively (Figure 4A). As expected from the negative results of the toxicity tests, liver histologies were normal (Figure 4B). We did not find EYFP-negative hepatocytes either in the liver parenchyma or around the portal veins (Figure 4, C-F). This result shows that all newly formed hepatocytes were derived from preexisting hepatocytes, which excludes a contribution of liver progenitor cells to normal liver homeostasis.

The origin of new hepatocytes after hepatocyte-specific injury. Next, we asked whether liver progenitor cells are involved in replacing hepatocytes lost due to acute injury. A single dose of CCl4 causes necrosis specifically in pericentral hepatocytes because they are the site of its metabolic activation (56). Within 2 days after intraperitoneal CCl4 injection, hepatocytes in the periportal and intermediate zones of the liver lobule begin to proliferate and replace the lost pericentral cells $(20,22)$. Liver progenitor cells were not believed to contribute to hepatocyte regeneration in this model of hepatocyte-specific injury until Furuyama et al. reported that a large fraction of new periportal hepatocytes derives from liver progenitor cells after acute CCl4 intoxication (18).

To test this surprising finding, we repeated the experiment described by Furuyama et al. in our hepatocyte fate-tracing model (Figure 5A). As expected, a single CCl4 injection caused marked hepatocyte necrosis around central veins within 2 days (Figure 5B and Supplemental Figure 5, A and B). In contrast to Furuyama et al., we did not detect any hepatocytes that were EYFP negative and thus not derived from preexisting hepatocytes (Figure 5, C and D, and Supplemental Figure 5C). However, when we injected CCl4 biweekly for 6 weeks, a chronic intoxication protocol used to induce liver fibrosis (51), we found that $1.3 \%$ of all hepatocytes were EYFP negative (Figure 6, A-C, Supplemental Figure 6A, and Supplemental Figure 7). Lack of EYFP in the presence of MUP expression indicated that these were mature hepatocytes that were not derived from preexisting hepatocytes (Figure 6D). Thus, these hepatocytes most likely originated from liver progenitor cells. Chronic CCl4 treatment has previously been reported to induce bile duct proliferation (57), which may explain why we found rare liver progenitor cellderived hepatocytes in mice after chronic, but not after acute, $\mathrm{CCl} 4$ intoxication. In accordance with a recently revised view on epithelial-mesenchymal transition (EMT) in liver fibrosis, we did not find hepatocyte-derived stellate cells (Supplemental Figure 6B and ref. 51). 
A
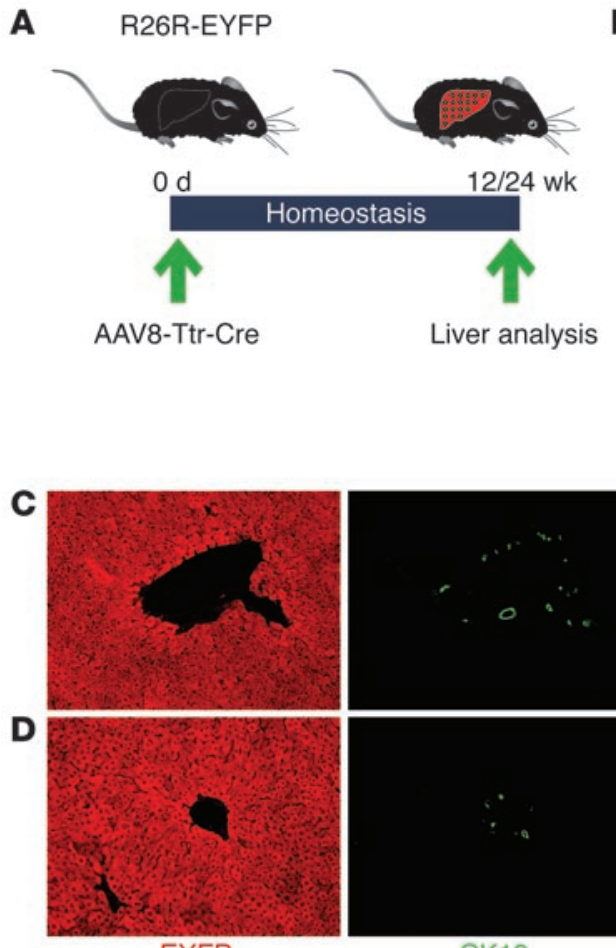

EYFP

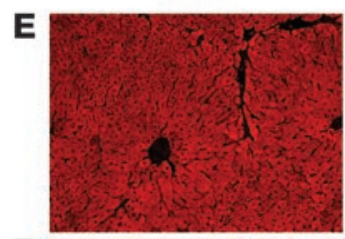

$\mathbf{F}$

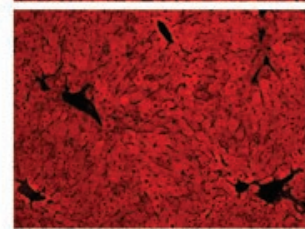

EYFP

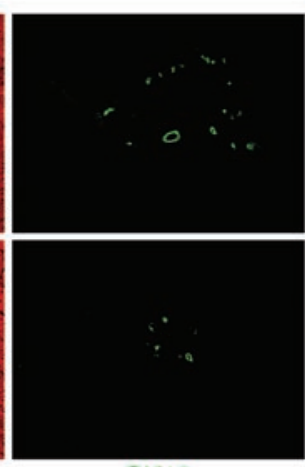

CK19
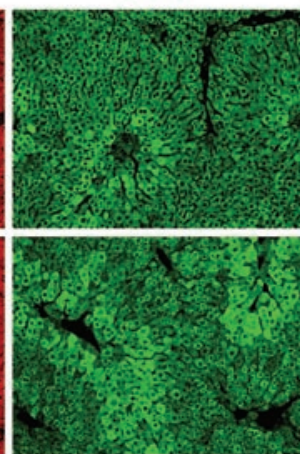

MUP
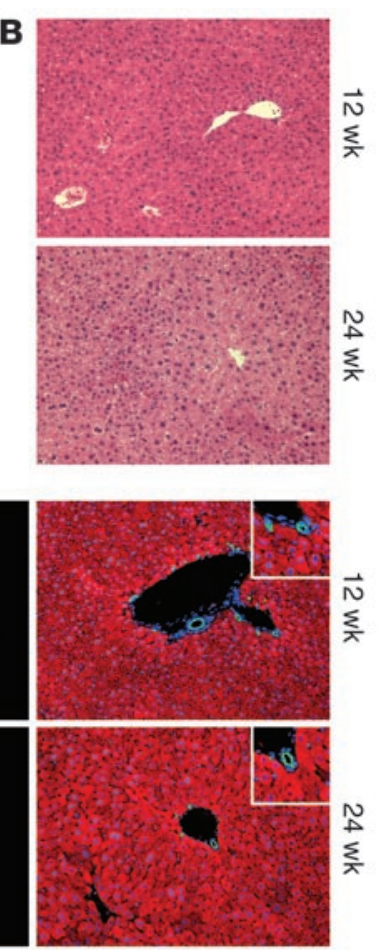

CK19 + EYFP + DAPI
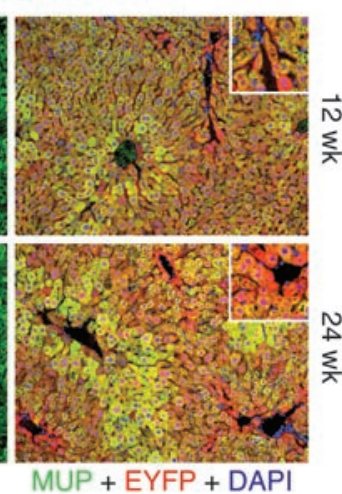

$M U P+E Y F P+D A P I$

\section{Figure 4}

Hepatocyte fate tracing in liver homeostasis. (A) Livers of adult R26R-EYFP mice were analyzed 12 and 24 weeks after injection of $4 \times 10^{11}$ viral genomes of AAV8-Ttr-Cre. (B) H\&E staining shows normal liver histologies. (C and D) Coimmunostaining for EYFP (red) and CK19 (green) shows normal hepatocyte plates and bile ducts 12 weeks (C) and 24 weeks (D) after AAV8-Ttr-Cre injection. No hepatocyte appears EYFP negative at both time points. ( $E$ and $F$ ) Coimmunostaining for EYFP (red) and MUP (green) confirms that all hepatocytes express EYFP 12 weeks $(E)$ and 24 weeks (F) after AAV8-Ttr-Cre injection. Nuclei were stained with DAPI (blue). Original magnification, $\times 100$, insets $\times 200$. 15 liver sections from 3 mice were analyzed for each experiment.

The origin of new hepatocytes after loss of intact liver lobes. We also investigated the role of liver progenitor cells in $2 / 3 \mathrm{PH}$, a model of hepatocyte regeneration not associated with hepatocyte injury and necrosis (5). Liver regeneration after $2 / 3 \mathrm{PH}$ has been shown to be mediated by proliferation of differentiated cells $(24,25)$. Hepatocytes enter the cell cycle first, followed by biliary epithelial cells and nonparenchymal cells. Virtually all residual hepatocytes contribute, and liver progenitor cells are not believed to be involved $(19,21,23)$. To formally test this concept, we per-

formed 2/3 PH in our hepatocyte fate-tracing model. To allow complete liver regeneration, including restoration of the normal lobular architecture, we analyzed the mice 3 weeks after the surgery (Figure 7, A and B). Surprisingly, we found that $1.4 \%$ of all hepatocytes were EYFP negative and could therefore not be derived from preexisting hepatocytes (Figure 7, C and D, and Supplemental Figure 7). These cells were located in close proximity to bile ducts, but were negative for CK19 and positive for MUP, indicating that they were fully mature hepatocytes (Figure 7, D and E). Our finding that liver progenitor cells contribute a small number of hepatocytes to liver regeneration after $2 / 3$ $\mathrm{PH}$ is in accordance with results recently reported by Furuyama et al. (18).

The origin of new biliary epithelial cells and liver progenitor cells after biliary injury. Recent studies showed that transplanted hepatocytes can give rise to biliary epithelial cells in rats after bile duct ligation (BDL), a model of biliary injury (26-29). This finding suggests that biliary injury induces conversion of hepatocytes into biliary epithelial cells, which we decided to test by performing BDL in our hepatocyte fate-tracing model (Figure 8A). Because ligation of the common bile duct has severe systemic effects in C57BL/6 mice, including hepatocyte injury, weight loss, and mortality, we used selective ligation of the left hepatic duct as a model of biliary-specific injury (58). As previously reported, affected liver lobes developed extensive ductular reactions that stained positive for CK19 within 10 days of BDL (Figure 8, B and C). Coexpression of CK19 and EYFP was readily detectable in biliary epithelial cells formed after BDL in Alb-Cre, R26R-EYFP control mice (Supplemental Figure 8A). In contrast, we did not detect cells that expressed both CK19 and EYFP in our hepatocyte fate-tracing model (Figure 8C). Thus, our results show that new biliary epithelial cells generated in response to BDL do not derive from hepatocytes in mice. We also ruled out that stellate cells, which massively expand after BDL (58), are the product of hepatocyte EMT (Supplemental Figure 8B).

It has not been reported whether conversion of hepatocytes into biliary epithelial cells in rats occurs directly or whether it entails dedifferentiation into liver progenitor cells followed by biliary differentiation. To determine whether liver progenitor cells emerging during biliary injury originate from hepatocytes, we treated our hepatocyte fate-tracing model with a diet containing the toxin 3,5-diethoxycarbonyl-1,4-dihydrocollidine (DDC) (Figure 9A, Supplemental Figure 9A, and ref. 59). DDC feeding activates liver progenitor cells in mice that correspond to oval cells in rats (60) and ductular reactions in humans (61).

Mouse liver progenitor cells have been shown to be bipotent, but the injury caused by DDC promotes predominantly biliary differentiation (9-11). Nevertheless, we found a small number of EYFPnegative cells expressing the hepatocyte-specific marker HNF $4 \alpha$ in our hepatocyte fate-tracing model after DDC feeding and also after BDL (Supplemental Figure 10, A-C). The cells were invariably located in periportal areas and most likely represent liver 
A

R26R-EYFP

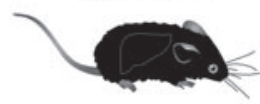

$0 \mathrm{~d}$

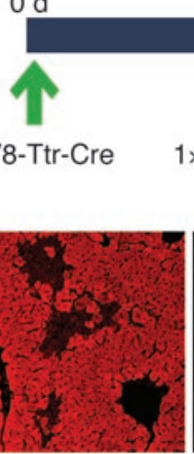

EYFP

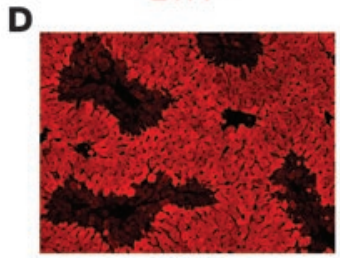

EYFP
B

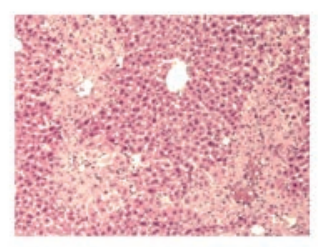

Liver analysis

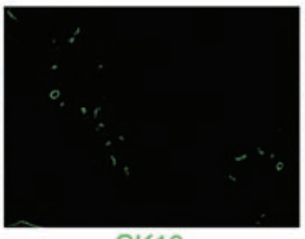

CK19

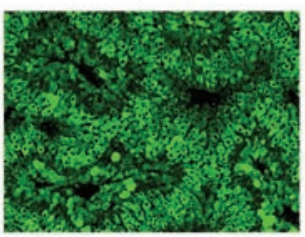

MUP

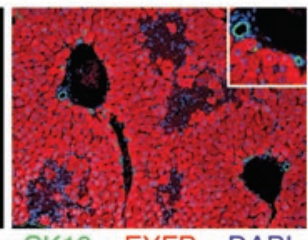

CK19 + EYFP + DAPI

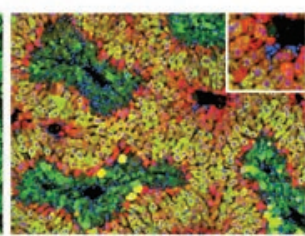

$M U P+E Y F P+D A P I$

\section{Figure 5}

Hepatocyte fate tracing after acute $\mathrm{CCl} 4$ intoxication. (A) Adult R26R-EYFP mice were injected with $4 \times 10^{11}$ viral genomes of AAV8-Ttr-Cre and received a single dose of $\mathrm{CCl} 47$ days later. Livers were analyzed 2 days after $\mathrm{CCl} 4$ intoxication. (B) H\&E staining shows pericentral hepatocyte necrosis. (C) Coimmunostaining for EYFP (red) and CK19 (green) confirms hepatocyte loss in pericentral areas, while periportal areas, including bile ducts, appear normal. (D) Coimmunostaining for EYFP (red) and MUP (green) fails to detect EYFP-negative hepatocytes emerging in periportal areas. Pericentral necrotic hepatocytes appear weakly MUP positive (Supplemental Figure $5 \mathrm{C}$ ). Nuclei were stained with DAPI (blue). Original magnification, $\times 100$, insets $\times 200.20$ liver sections from 4 mice were analyzed. progenitor cells that committed to the hepatocyte fate. However, the vast majority of cells in the ductular reactions, which progressively expanded into the parenchyma with time on the DDC diet, expressed CK19, indicating that they were liver progenitor cells or biliary epithelial cells (Figure 9, B-D). As expected, all cells positive for CK19 were also positive for EYFP in DDC-treated Alb-Cre, R26R-EYFP control mice (Supplemental Figure 9B). In contrast, none of the CK19-positive cells coexpressed EYFP in our hepato- cyte fate-tracing model (Figure 9, C and D). These results show that DDC-induced liver progenitor cells and their differentiated progeny are not derived from hepatocytes.

\section{Discussion}

Recent findings have rekindled an old debate about whether hepatocytes or liver progenitor cells are the primary source of new hepatocytes in liver homeostasis and regeneration.
A R26R-EYFP

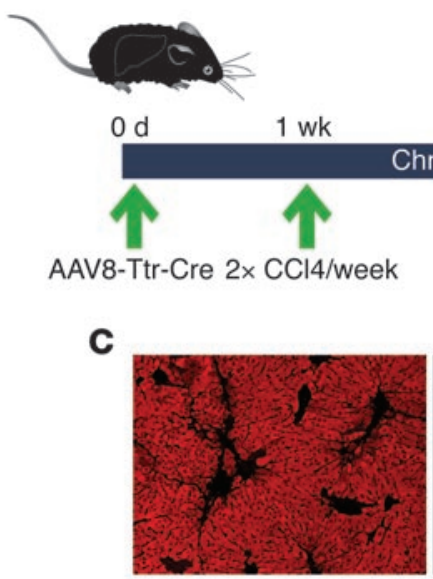

D

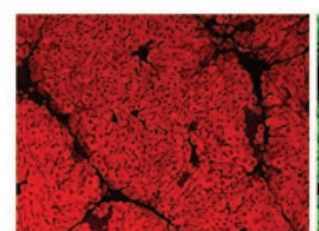

EYFP

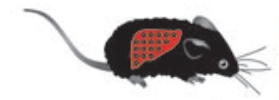

7 wk
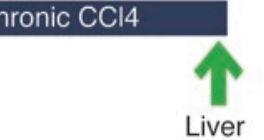

analysis

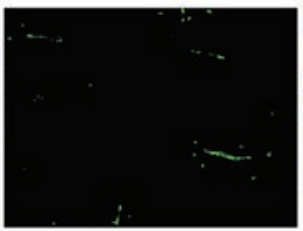

CK19

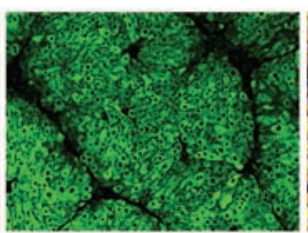

MUP
B
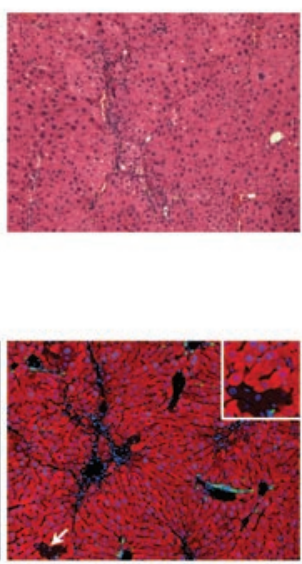

CK19 + EYFP + DAPI

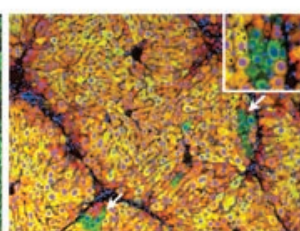

$M U P$ + EYFP + DAPI 
A R26R-EYFP

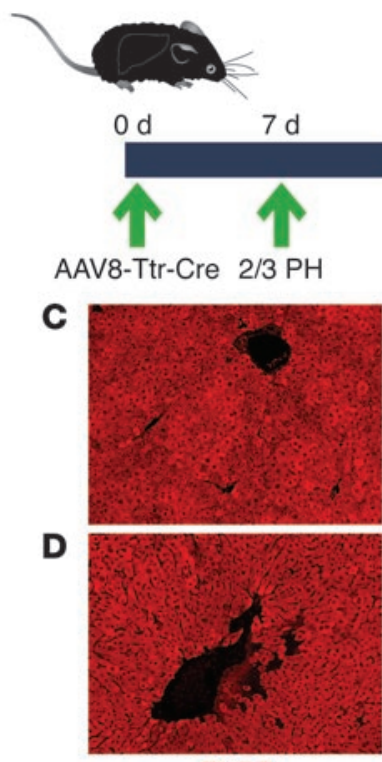

EYFP

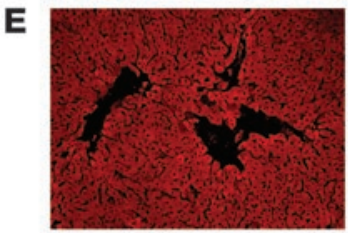

EYFP

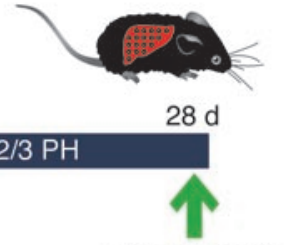

Liver analysis

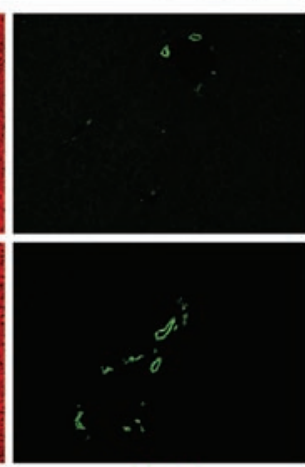

CK19

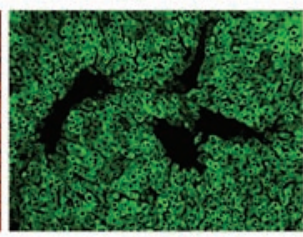

MUP
B
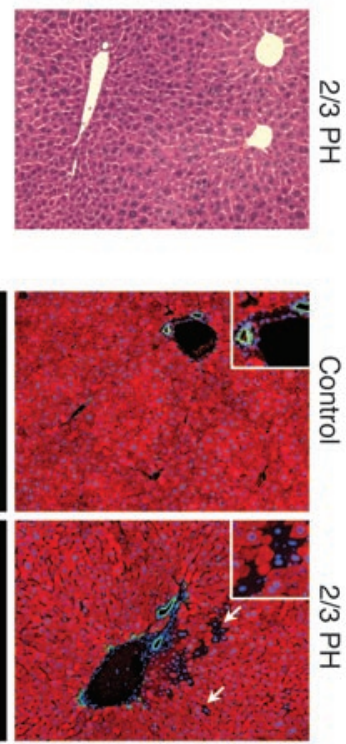

CK19 + EYFP + DAPI

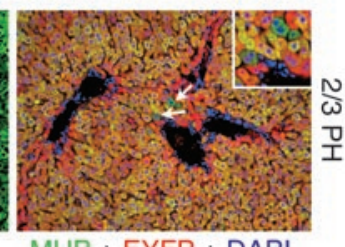

\section{Figure 7}

Hepatocyte fate tracing after $2 / 3 \mathrm{PH}$. (A) Adult R26REYFP mice were injected with $4 \times 10^{11}$ viral genomes of AAV8-Ttr-Cre and underwent 2/3 PH 7 days later. Livers were analyzed 21 days after $2 / 3 \mathrm{PH}$. Liver lobes removed by $2 / 3 \mathrm{PH}$ were used as controls. (B) H\&E staining shows restoration of normal liver histology 21 days after 2/3 $\mathrm{PH}$. (C and $\mathbf{D})$ Coimmunostaining for EYFP (red) and CK19 (green) shows that all hepatocytes expressed EYFP before 2/3 PH (C) but that clusters of EYFP-negative cells resembling hepatocytes are present in periportal areas after $2 / 3$ $\mathrm{PH}$ (D, white arrows). (E) Coimmunostaining for EYFP (red) and MUP (green) confirms the presence of single cells and clusters of EYFP-negative, MUP-positive hepatocytes (white arrows). Nuclei were stained with DAPI (blue). Original magnification, $\times 100$, insets $\times 200$. 20 liver sections from 4 mice were analyzed.
Hepatocytes are likely candidates because they have stem celllike proliferative capabilities $(7,8)$, which make liver progenitor cells dispensable. However, data obtained with new mouse models (18) and refined analyses of human livers (17) suggest that liver progenitor cells contribute significantly, if not predominantly, to replacing hepatocytes during normal turnover and after acute injury. Other recent reports have raised the possibility of lineage conversion of hepatocytes in response to biliary injury; the data suggest that hepatocyte differentiation is not fixed, but that hepatocytes can supply new biliary epithelial cells when the regenerative capabilities of both biliary epithelial cells and liver progenitor cells are impaired (26-29).

To test these new findings, we generated an in situ hepatocyte fate-tracing model based on rapid and specific marker gene activation in all hepatocytes of adult R26R-EYFP mice with the nontoxic vector AAV8-Ttr-Cre. We found that all newly formed hepatocytes in the normal adult liver are derived from preexisting hepatocytes. Our result contradicts the finding by Furuyama et al. that liver progenitor cells are primarily responsible for liver homeostasis (18) and supports the previous paradigm of hepatocyte self duplication as the principal mechanism. While we can only speculate on the reason for the finding by Furuyama et al., possibly their experimental system, which was based on induction of Cre expression in liver progenitor cells and biliary epithelial cells in adult mice, caused toxicity and liver progenitor cell activation. An unrecognized environmental factor may have subsequently perpetuated liver progenitor cell activation by causing subclinical chronic hepatocyte injury. This hypothetical, but not unlikely, scenario would explain why homeostatic hepatocyte replacement occurred in their mice in the form of bridging between periportal areas, a characteristic feature of liver progenitor cell-mediated repair of chronic hepatocyte injury $(18,49)$.

We also used our hepatocyte fate-tracing model to identify the origin of new hepatocytes in liver regeneration. In accordance with findings by Furuyama et al. (18), we found that liver progenitor cells contribute to hepatocyte replacement after $2 / 3$ $\mathrm{PH}$ and $\mathrm{CCl} 4$ intoxication. This finding is surprising because hepatocytes lost due to these nontoxic or hepatocyte-specific insults were previously believed to be replaced only by self duplication of residual hepatocytes (19-23). However, our results also show that the number of hepatocytes derived from liver progenitor cells after $2 / 3 \mathrm{PH}$ or $\mathrm{CCl} 4$ intoxication is very small and thus most likely irrelevant. Moreover, we found that chronic, but not acute, CCl4 intoxication produces liver progenitor cell-derived hepatocytes. These results contradict the finding of Furuyama et al. that acute $\mathrm{CCl} 4$ intoxication leads to rapid production of a substantial number of hepatocytes by liver progenitor cells (18). Viewed together, our findings suggest that bile duct proliferation, which is triggered by both chronic CCl4 intoxication (57) and $2 / 3 \mathrm{PH}(24)$, is a prerequisite for the emergence of liver progenitor cell-derived hepatocytes.

Finally, we failed to find hepatocyte-derived biliary epithelial cells in our hepatocyte fate-tracing model. Our results obtained with BDL argue against the recently proposed concept of biliary injury causing conversion of hepatocytes into biliary epithelial cells (26-29). As was done in the original studies $(26,28,29)$, we identified biliary epithelial cells based on CK19 expression. However, differences also exist between our experiments and the 
A R26R-EYFP

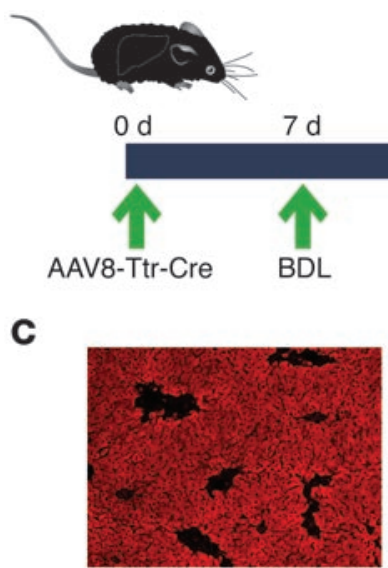

EYFP

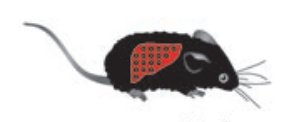
BDL

$17 \mathrm{~d}$

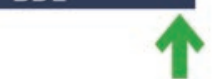

Liver

analysis
B
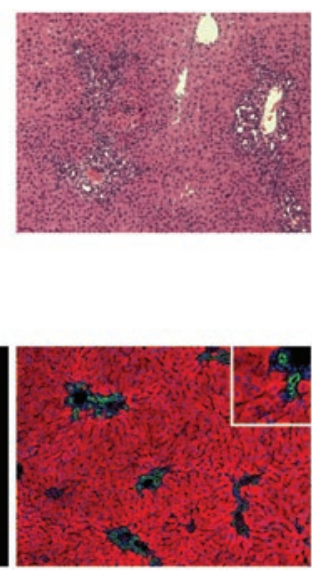

CK19 + EYFP + DAPI

\section{Figure 8}

Hepatocyte fate tracing after BDL. (A) Adult R26R-EYFP mice were injected with $4 \times 10^{11}$ viral genomes of AAV8-Ttr-Cre and underwent $B D L 7$ days later. Livers were analyzed 10 days after BDL. (B) H\&E staining shows extensive ductular reactions. (C) Coimmunostaining for EYFP (red) and CK19 (green) shows no double-positive cells. Nuclei were stained with DAPI (blue). Original magnification, $\times 100$, insets $\times 200$. 25 liver sections from 5 mice were analyzed. original ones that may warrant further investigation. First, we used in situ hepatocyte fate tracing in mice, whereas the original studies used hepatocyte transplantation into rats. Although unlikely, transplanted rat hepatocytes may be more amenable to lineage conversion than resident mouse hepatocytes. Second, we did not combine BDL with biliary toxins. A study in rats found that, while at least $1.75 \%$ of all biliary epithelial cells were hepatocyte derived after BDL alone, the frequency could be markedly increased by additional exposure to methylene dianiline (DAPM), a biliary toxin that blocks proliferation of biliary epithelial cells and liver progenitor cells (27). Although little information is available on the effects of DAPM in mice $(62,63)$, combining BDL with DAPM intoxication may block proliferation of both biliary epithelial cells and liver progenitor cells in these animals as well. Nevertheless, we are confident that our system would have detected even a very small number of hepatocyte-derived biliary epithelial cells, considering that we could identify rare liver progenitor cell-derived hepatocytes after $2 / 3 \mathrm{PH}$ or chronic CCl4 intoxication. Third, our BDL protocol entailed ligation of the left hepatic duct for 10 days, not the common bile duct for 30 days, as in the rat studies. We chose this protocol because it avoids the severe systemic effects of common BDL in C57BL/6 mice, including hepatocyte injury, weight loss, and mortality, while causing indistinguishable ductular reactions in the affected liver lobes (58). Therefore, we cannot rule out that by avoiding injury and death of hepatocytes, we may have limited compensatory hepatocyte proliferation, which is generally viewed as a promoter of lineage conversion (32). On the other hand, recent studies showed

\section{Figure 9}

Hepatocyte fate tracing after DDC feeding. (A) Adult R26REYFP mice were injected with $4 \times 10^{11}$ viral genomes of AAV8-Ttr-Cre, and DDC feeding began 1 week later. Livers were analyzed after 3 or 8 weeks of DDC feeding. (B) H\&E staining shows ductular reactions expanding with time of DDC feeding. (C and D) Coimmunostaining for EYFP (red) and CK19 (green) shows no doublepositive cells after 3 (C) or 8 (D) weeks of DDC feeding. Nuclei were stained with DAPI (blue). Original magnification, $\times 100$, insets $\times 200.20$ liver sections from 4 mice were analyzed for each experiment.
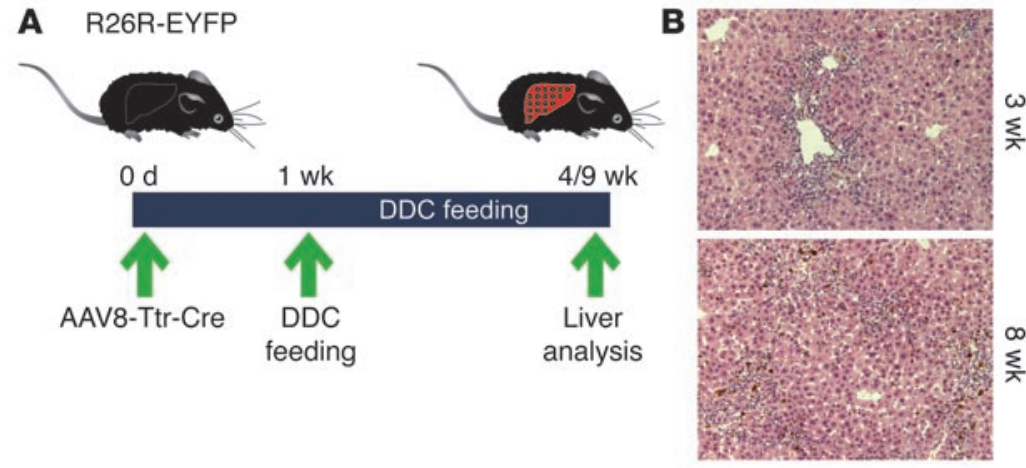

C
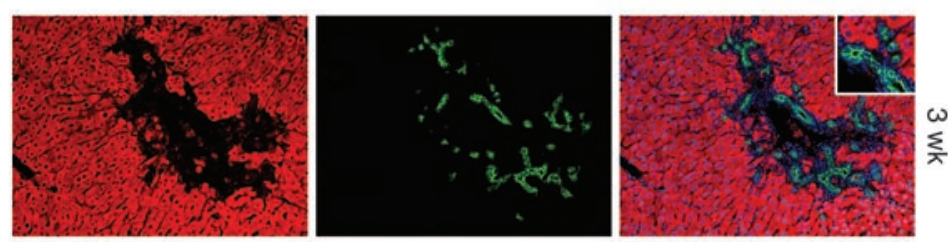

D
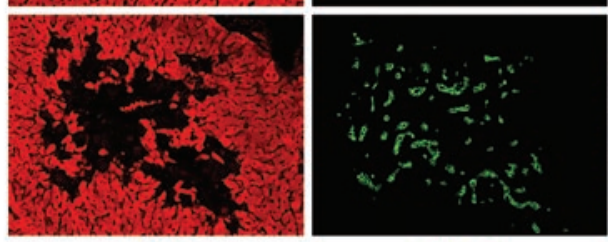

EYFP

CK19

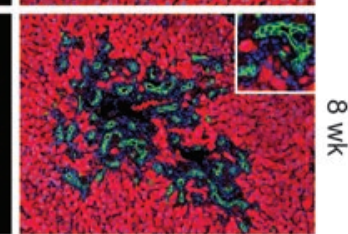

CK19 + EYFP + DAPI 
that proliferation is not needed for lineage conversion if the cell types are developmentally related, such as pancreatic exocrine and endocrine cells (64), B cells and macrophages (65), or, in this case, hepatocytes and biliary epithelial cells.

Hepatocyte-derived biliary epithelial cells were also absent in our hepatocyte fate-tracing model after DDC feeding. Because most DDC-induced liver progenitor cells express CK19 (10, 11, 66), our results are consistent with previous findings that transplanted hepatocytes are not precursors of liver progenitor cells in mice (66). Nevertheless, it is in principle possible that our analysis missed rare hepatocyte-derived liver progenitor cells that were not expressing CK19. However, if such cells were present, it would be surprising that none of them differentiated into CK19-positive biliary epithelial cells during the extensive expansion of ductular reactions triggered by 8 -week-long DDC feeding.

In conclusion, our study settles a long-standing debate by demonstrating that liver homeostasis is mediated by self duplication of mature hepatocytes and does not involve liver progenitor cells. Moreover, we show that liver progenitor cells contribute only minimally to regeneration of acutely lost hepatocytes. Thus, our results support the view that liver progenitor cells provide a backup system for injury states in which the proliferative capabilities of hepatocytes are impaired. Our findings underscore the importance of hepatocytes for liver regeneration and suggest that harnessing liver progenitor cells for therapy will require knowing the signals that trigger their activation. We also used our hepatocyte fate-tracing model to test the emerging concept that hepatocytes spontaneously convert into biliary epithelial cells in states of biliary injury. We found no evidence for hepatocyte-derived biliary epithelial cells or liver progenitor cells in 2 commonly used models of biliary injury. In the future, hepatocyte fate tracing could be used to determine whether hepatocyte lineage conversion can be forced by overexpression of biliary-specific transcription factors. Hepatocyte fate tracing could also serve to advance our understanding of hepatocarcinogenesis by determining whether liver cancers exhibiting liver progenitor cell characteristics derive from dedifferentiated hepatocytes or liver progenitor cells. In addition, AAV8-Ttr-Cre could be generally useful for liver research by facilitating timed gene inactivation in all hepatocytes, but no other liver cells, of conditional knockout mice.

\section{Methods}

Supplemental Methods are available online with this article.

Mice. 8- to 10-week-old homozygous R26R mice (34), homozygous R26REYFP mice (45), and mice homozygous for R26R-EYFP and heterozygous for Alb-Cre (47) were used. All mice were on the C57BL/6 strain background and kept under barrier conditions. All procedures were approved by the Institutional Animal Care and Use Committee at UCSF.

AAV8-Ttr-Cre construction and production. The sequences of the Ttr promoter and Cre recombinase were PCR amplified from the plasmids TTRXpo-5 (67) and pML78 (43), respectively. A Ttr-Cre expression cassette was constructed to replace the $\mathrm{H} 1$ promoter in the double-stranded vector plasmid dsAAV-H1 (42). The dsAAV-H1 backbone is based on the AAV2 genome, but contains heterologous packaging signals from AAV2 and AAV4 to prevent inactivating recombination during propagation. For AAV8-Ttr-Cre production, A293 cells were transfected with the newly generated plasmid dsAAV-Ttr-Cre, the adenoviral helper plasmid PVAE2AE4-5 (68), and the AAV8 capsid expression plasmid p5E18-VD2/8 (69) using the calcium phosphate method (42). Virus was collected 72 hours after transfection and purified by centrifugation on a density gradient of cesium chloride (Invitrogen).

AAV8-Ttr-Cre titration. The vector titer was determined by dot blot analysis as described (42) and qPCR. For dot blot analysis, serial $\times 10$ dilutions of the vector were spotted onto a nylon membrane next to a standard consisting of serial $\times 2$ dilutions of the linearized original vector plasmid (Supplemental Figure 1A). Signals were detected by using a ${ }^{32} \mathrm{P}-$ labeled probe against the Ttr promoter sequence and quantified using a phosphoscreen and Quantity One software (Bio-Rad). For qPCR titration, alkaline lysis was performed to release viral DNA from AAV particles: $10 \mu \mathrm{l}$ of vector was incubated with $10 \mu \mathrm{l} \mathrm{TE}(10 \mathrm{mM}$ Tris-HCl $\mathrm{pH} 7.5,1 \mathrm{mM}$ EDTA) and $20 \mu \mathrm{l} 2 \mathrm{M} \mathrm{NaOH}$ for 30 minutes at $56^{\circ} \mathrm{C}$, followed by neutralization with $38 \mu 11 \mathrm{M} \mathrm{HCl}$ and $922 \mu 1 \mathrm{H}_{2} \mathrm{O}$. qPCR reactions were set up in $10 \mu \mathrm{l} 1 \times$ SensiMix II Probe (Bioline) containing $0.4 \mu \mathrm{M}$ primer and $0.1 \mu \mathrm{M}$ probe, and subjected to 40 cycles of amplification ( 2 steps per cycle: $95^{\circ} \mathrm{C}$ for 10 seconds and $60^{\circ} \mathrm{C}$ for 20 seconds). All reactions were set up in triplicate and run in a Rotor-Gene 6000 system (QIAGEN) using the following primers and probe: forward, TGTTCCGATACTCTAATCTCCC; reverse, TATACCCССТCCTTCCAACC; probe, FAM-TTTGGAGTCAGCTTGGCAGGGATCA-BHQ1 (MWG Biotech). Titers were calculated based on a standard curve (generated with the original vector plasmid containing the Ttr promoter) using the RotorGene 6000 software (Supplemental Figure 1B).

AAV8-Ttr-Cre injection. The purified vector was diluted in PBS. The tailvein injection volume was limited to $100 \mu \mathrm{l}$ to avoid hydrodynamic effects.

Liver function tests. Plasma was diluted $4 \times$ in $0.9 \%$ sodium chloride and assayed for alanine (ALT), aspartate (AST) transaminase, direct bilirubin, and albumin with an ADVIA 1800 (Siemens) chemistry analyzer.

CCl4 intoxication. For acute intoxication, a single dose of $0.5 \mu \mathrm{l} / \mathrm{g}$ body weight CCl4 diluted $4 \times$ in corn oil (both Sigma-Aldrich) was injected intraperitoneally (18). For chronic intoxication, the same dose was injected twice weekly for 6 weeks.

$2 / 3 \mathrm{PH}$. With mice under isoflurane anesthesia and sterile conditions, two-thirds of the liver was surgically removed as previously described (70).

$B D L$. With mice under isoflurane anesthesia and sterile conditions, the left hepatic duct was ligated as previously described (58).

DDC feeding. Mice received mouse diet 5015 (TestDiet) containing $0.1 \%$ w/w DDC (Sigma-Aldrich).

Immunostaining. Tissue samples were fixed overnight in neutral-buffered formalin containing zinc (Anatech), embedded in paraffin, cut into $5-\mu \mathrm{m}-$ thick sections, and placed on Superfrost Plus slides (Fisher Scientific). Sections were deparaffinized and incubated in boiling Antigen Retrieval Citra Solution (BioGenex) for 10 minutes. After cooling down, sections were blocked in $10 \%$ serum for 1 hour and then incubated with primary antibodies overnight at $4{ }^{\circ} \mathrm{C}$ and secondary antibodies for 1 hour at room temperature (Supplemental Tables 1 and 2). Nuclear DNA was stained with 300 nM DAPI (Millipore).

qRT-PCR. RNA was isolated with the TRIzol kit (Invitrogen). Reverse transcription was performed using qScript cDNA Supermix (Quanta Biosciences). qPCR was performed using SYBR green reagent (Quanta Biosciences) in a ViiA 7 system (Applied Biosystems). Reactions were performed twice in triplicate. Expression was normalized to Gapdh and quantified using the $2-\Delta \Delta \mathrm{Ct}$ method.

Statistics. Data are expressed as mean \pm SEM. Statistical significance was determined by 2-way ANOVA followed by 2 -tailed Student's $t$ test. $P<0.05$ was considered significant.

\section{Acknowledgments}

This work was supported by a California Institute for Regenerative Medicine New Faculty Award (RN2-00950) to H. Willenbring 
and by the UCSF Liver Center (P30 DK026743). R. Ng was supported by a scholarship from the Agency of Science, Technology and Research (A*STAR), Singapore. The authors thank Donghui Wang for tail-vein injections, John P. Morris IV for help with EYFP immunostainings, Craig Dorrell, Andrew Pierce, and Amanda Goodsell for help with FACS, and Pamela Derish and Alexis Bailey for editing the manuscript.

1. Fausto N. Liver regeneration and repair: hepatocytes, progenitor cells, and stem cells. Hepatology. 2004;39(6):1477-1487.

2. Fausto N, Campbell JS, Riehle KJ. Liver regeneration. Hepatology. 2006;43(2 Suppl 1):S45-53.

3. Michalopoulos GK. Liver regeneration. J Cell Physiol. 2007;213(2):286-300

4. Duncan AW, Dorrell C, Grompe M. Stem cells and liver regeneration. Gastroenterology. 2009; 137(2):466-481.

5. Michalopoulos GK. Liver regeneration: alternative epithelial pathways. Int J Biochem Cell Biol. 2011; 43(2):173-179.

6. Riehle KJ, Dan YY, Campbell JS, Fausto N. New concepts in liver regeneration. $J$ Gastroenterol Hepatol. 2011;26 suppl 1:203-212.

7. Overturf K, al-Dhalimy M, Ou CN, Finegold M, Grompe M. Serial transplantation reveals the stem-cell-like regenerative potential of adult mouse hepatocytes. Am J Pathol. 1997;151(5):1273-1280.

8. Overturf K, Al-Dhalimy M, Finegold M, Grompe M The repopulation potential of hepatocyte populations differing in size and prior mitotic expansion. Am J Pathol. 1999;155(6):2135-2143.

9. Sackett SD, et al. Foxl1 is a marker of bipotential hepatic progenitor cells in mice. Hepatology. 2009; 49(3):920-929.

10. Shin S, et al. Foxl1-Cre-marked adult hepatic progenitors have clonogenic and bilineage differentiation potential. Genes Dev. 2011;25(11):1185-1192.

11. Dorrell C, et al. Prospective isolation of a bipotential clonogenic liver progenitor cell in adult mice. Genes Dev. 2011;25(11):1193-1203.

12. Zajicek G, Oren R, Weinreb M Jr. The streaming liver. Liver. 1985;5(6):293-300

13. Fellous TG, et al. Locating the stem cell niche and tracing hepatocyte lineages in human liver. Hepatology. 2009;49(5):1655-1663.

14. Bralet MP, Branchereau S, Brechot C, Ferry N. Cell lineage study in the liver using retroviral mediated gene transfer. Evidence against the streaming of hepatocytes in normal liver. Am J Pathol. 1994; 144(5):896-905.

15. Kennedy S, Rettinger S, Flye MW, Ponder KP. Experiments in transgenic mice show that hepatocytes are the source for postnatal liver growth and do not stream. Hepatology. 1995;22(1):160-168.

16. Lowes KN, Brennan BA, Yeoh GC, Olynyk JK. Oval cell numbers in human chronic liver diseases are directly related to disease severity. Am J Pathol. 1999; 154(2):537-541.

17. Schmelzer E, et al. Human hepatic stem cells from fetal and postnatal donors. J Exp Med. 2007; 204(8):1973-1987.

18. Furuyama K, et al. Continuous cell supply from a Sox9-expressing progenitor zone in adult liver, exocrine pancreas and intestine. Nat Genet. 2011; 43(1):34-41.

19. Bucher NL, Swaffield MN. The rate of incorporation of labeled thymidine into the deoxyribonucleic acid of regenerating rat liver in relation to the amount of liver excised. Cancer Res. 1964 . 24:1611-1625.

20. Morrison GR, Brock FE, Karl IE, Shank RE. Quantitative analysis of regenerating and degenerating areas within the lobule of the carbon tetrachloride-injured liver. Arch Biochem Biophys. 1965; 111(2):448-460.
Received for publication June 1, 2011, and accepted in revised form October 12, 2011.

Address correspondence to: Holger Willenbring, University of California San Francisco, 35 Medical Center Way, Campus Box 0665, San Francisco, California 94143, USA. Phone: 415.476.2417; Fax: 415.514.2346; E-mail: willenbringh@stemcell.ucsf.edu.
21. Stocker E, Heine WD. Regeneration of liver parenchyma under normal and pathological conditions. Beitr Pathol. 1971;144(4):400-408.

22. Sell S. Heterogeneity and plasticity of hepatocyte lineage cells. Hepatology. 2001;33(3):738-750.

23. Fausto N, Campbell JS. The role of hepatocytes and oval cells in liver regeneration and repopulation. Mech Dev. 2003;120(1):117-130.

24. Grisham JW. A morphologic study of deoxyribonucleic acid synthesis and cell proliferation in regenerating rat liver; autoradiography with thymidine- $\mathrm{H} 3$. Cancer Res. 1962;22:842-849.

25. Michalopoulos GK, DeFrances MC. Liver regeneration. Science. 1997;276(5309):60-66.

26. Fukuda K, et al. The origin of biliary ductular cells that appear in the spleen after transplantation of hepatocytes. Cell Transplant. 2004;13(1):27-33.

27. Michalopoulos GK, Barua L, Bowen WC. Transdifferentiation of rat hepatocytes into biliary cells after bile duct ligation and toxic biliary injury. Hepatology. 2005;41(3):535-544.

28. Watanabe $\mathrm{H}$, et al. Transdifferentiation into biliary ductular cells of hepatocytes transplanted into the spleen. Pathology. 2008;40(3):272-276.

29. Limaye PB, Bowen WC, Orr A, Apte UM, Michalopoulos GK. Expression of hepatocytic- and biliaryspecific transcription factors in regenerating bile ducts during hepatocyte-to-biliary epithelial cell transdifferentiation. Comp Hepatol. 2010;9:9.

30. Crosby HA, et al. Immunolocalization of putative human liver progenitor cells in livers from patients with end-stage primary biliary cirrhosis and sclerosing cholangitis using the monoclonal antibody OV-6. Am J Pathol. 1998;152(3):771-779.

31. Limaye PB, et al. Expression of specific hepatocyte and cholangiocyte transcription factors in human liver disease and embryonic development. Lab Invest. 2008;88(8):865-872.

32. Zhou Q, Melton DA. Extreme makeover: converting one cell into another. Cell Stem Cell. 2008; 3(4):382-388.

33. Alison MR, Lin WR. Hepatocyte turnover and regeneration: Virtually a virtuoso performance. Hepatology. 2011;53(4):1393-1396.

34. Soriano P. Generalized lacZ expression with the ROSA26 Cre reporter strain. Nat Genet. 1999; 21(1):70-71.

35. Imai T, Chambon P, Metzger D. Inducible site-specific somatic mutagenesis in mouse hepatocytes. Genesis. 2000;26(2):147-148.

36. Tannour-Louet M, Porteu A, Vaulont S, Kahn A Vasseur-Cognet M. A tamoxifen-inducible chimeric Cre recombinase specifically effective in the fetal and adult mouse liver. Hepatology. 2002; 35(5):1072-1081.

37. Schuler M, Dierich A, Chambon P, Metzger D. Efficient temporally controlled targeted somatic mutagenesis in hepatocytes of the mouse. Genesis. 2004; 39(3):167-172.

38. Kistner A, et al. Doxycycline-mediated quantitative and tissue-specific control of gene expression in transgenic mice. Proc Natl Acad Sci U S A. 1996; 93(20):10933-10938.

39. Rohlmann A, Gotthardt M, Hammer RE, Herz J. Inducible inactivation of hepatic LRP gene by cremediated recombination confirms role of LRP in clearance of chylomicron remnants. JClin Invest. 1998; 101(3):689-695.
40. Manickan E, Satoi J, Wang TC, Liang TJ. Conditional liver-specific expression of simian virus $40 \mathrm{~T}$ antigen leads to regulatable development of hepatic neoplasm in transgenic mice. J Biol Chem. 2001; 276(17):13989-13994.

41. Schonig K, Schwenk F, Rajewsky K, Bujard H. Stringent doxycycline dependent control of CRE recombinase in vivo. Nucleic Acids Res. 2002;30(23):e134.

42. Grimm D, et al. Fatality in mice due to oversaturation of cellular microRNA/short hairpin RNA pathways. Nature. 2006;441(7092):537-541.

43. Lewandoski M, Meyers EN, Martin GR. Analysis of Fgf8 gene function in vertebrate development. Cold Spring Harb Symp Quant Biol. 1997;62:159-168.

44. Nakai H, Fuess S, Storm TA, Muramatsu S, Nara Y, Kay MA. Unrestricted hepatocyte transduction with adeno-associated virus serotype 8 vectors in mice. J Virol. 2005;79(1):214-224.

45. Srinivas $\mathrm{S}$, et al. Cre reporter strains produced by targeted insertion of EYFP and ECFP into the ROSA26 locus. BMC Dev Biol. 2001;1:4.

46. Derman E. Isolation of a cDNA clone for mouse urinary proteins: age- and sex-related expression of mouse urinary protein genes is transcriptionally controlled. Proc Natl Acad Sci U S A. 1981; 78(9):5425-5429.

47. Postic C, Magnuson MA. DNA excision in liver by an albumin-Cre transgene occurs progressively with age. Genesis. 2000;26(2):149-150.

48. Xu X, et al. Induction of intrahepatic cholangiocellular carcinoma by liver-specific disruption of Smad4 and Pten in mice. J Clin Invest. 2006; 116(7):1843-1852.

49. Song G, et al. MicroRNAs control hepatocyte proliferation during liver regeneration. Hepatology. 2010; 51(5):1735-1743.

50. Willenbring $\mathrm{H}$, et al. Myelomonocytic cells are sufficient for therapeutic cell fusion in liver. Nat Med.2004; 10(7):744-748.

51. Taura K, et al. Hepatocytes do not undergo epithelial-mesenchymal transition in liver fibrosis in mice. Hepatology. 2010;51(3):1027-1036.

52. Scholten D, et al. Genetic labeling does not detect epithelial-to-mesenchymal transition of cholangiocytes in liver fibrosis in mice. Gastroenterology. 2010; 139(3):987-998.

53. Laitinen L. Griffonia simplicifolia lectins bind specifically to endothelial cells and some epithelial cells in mouse tissues. Histochem J. 1987; 19(4):225-234.

54. Magami Y, et al. Cell proliferation and renewal of normal hepatocytes and bile duct cells in adult mouse liver. Liver. 2002;22(5):419-425.

55. Macdonald RA. "Lifespan" of liver cells. Autoradiographic study using tritiated thymidine in normal, cirrhotic, and partially hepatectomized rats. Arch Intern Med. 1961;107:335-343.

56. Slater TF. Free-radical mechanisms in tissue injury. Biochem J. 1984;222(1):1-15.

57. Farazi PA, Zeisberg M, Glickman J, Zhang Y, Kalluri R, DePinho RA. Chronic bile duct injury associated with fibrotic matrix microenvironment provokes cholangiocarcinoma in p53-deficient mice. Cancer Res. 2006;66(13):6622-6627.

58. Wang B, et al. Role of alphavbeta6 integrin in acute biliary fibrosis. Hepatology. 2007;46(5):1404-1412.

59. Preisegger KH, Factor VM, Fuchsbichler A, Stumptner C, Denk H, Thorgeirsson SS. Atypical ductular 
proliferation and its inhibition by transforming growth factor beta1 in the 3,5-diethoxycarbonyl1,4-dihydrocollidine mouse model for chronic alcoholic liver disease. Lab Invest. 1999;79(2):103-109.

60. Tatematsu M, Ho RH, Kaku T, Ekem JK, Farber E. Studies on the proliferation and fate of oval cells in the liver of rats treated with 2-acetylaminofluorene and partial hepatectomy. Am J Pathol. 1984; 114(3):418-430.

61. Demetris AJ, Seaberg EC, Wennerberg A, Ionellie J, Michalopoulos G. Ductular reaction after submassive necrosis in humans. Special emphasis on analysis of ductular hepatocytes. Am J Pathol. 1996; 149(2):439-448.

62. Koniaris LG, Zimmers-Koniaris T, Hsiao EC, Chavin K, Sitzmann JV, Farber JM. Cytokine-responsive
gene-2/IFN-inducible protein-10 expression in multiple models of liver and bile duct injury suggests a role in tissue regeneration. J Immunol. 2001; 167(1):399-406.

63. Suzuki A, et al. Clonal identification and characterization of self-renewing pluripotent stem cells in the developing liver. J Cell Biol. 2002;156(1):173-184.

64. Zhou Q, Brown J, Kanarek A, Rajagopal J, Melton DA. In vivo reprogramming of adult pancreatic exocrine cells to beta-cells. Nature. 2008; 455(7213):627-632.

65. Xie H, Ye M, Feng R, Graf T. Stepwise reprogram ming of B cells into macrophages. Cell. 2004; 117(5):663-676.

66. Wang X, Foster M, Al-Dhalimy M, Lagasse E, Finegold M, Grompe M. The origin and liver repopulat- ing capacity of murine oval cells. Proc Natl Acad Sci US A. 2003;100(suppl 1):11881-11888.

67. Grimm D, et al. Argonaute proteins are key determinants of RNAi efficacy, toxicity, and persistence in the adult mouse liver. J Clin Invest. 2010; 120(9):3106-3119.

68. Matsushita T, et al. Adeno-associated virus vectors can be efficiently produced without helper virus. Gene Ther. 1998;5(7):938-945.

69. Gao GP, Alvira MR, Wang L, Calcedo R, Johnston J, Wilson JM. Novel adeno-associated viruses from rhesus monkeys as vectors for human gene therapy. Proc Natl Acad Sci U S A. 2002;99(18):11854-11859. 70. Mitchell C, Willenbring $\mathrm{H}$. A reproducible and well-tolerated method for $2 / 3$ partial hepatectomy in mice. Nat Protoc. 2008;3(7):1167-1170 